\title{
Attentional Cueing in Serious Games
}

\author{
Erik D. van der Spek, Herre van Oostendorp, Pieter Wouters, Laurens Aarnoudse \\ Department of Information and Computing Sciences \\ Utrecht University \\ Utrecht, The Netherlands \\ \{eriks, herre, pieterw\}@cs.uu.nl
}

\begin{abstract}
Games, and serious games especially, revolve around learning new material and integrating this into a mental model. However, playing games can be cognitively demanding and novices may fail to distinguish between relevant and irrelevant information. To overcome this, one can try to subtly cue the attention of the player to the relevant material. We empirically tested the use of auditory cues to guide the player in learning the correct procedure in a 3D serious triage training game. Learning did not significantly improve in the auditory cueing condition and mental model construction was even significantly worse in the cueing condition, as compared to a control group with no cues. Implications for game design and future research are subsequently discussed.
\end{abstract}

Keywords: serious games, cognitive load, cueing, learning, instructional design, game design, triage, games for health

\section{INTRODUCTION}

All games incorporate learning. Good games constantly throw up hurdles and obstacles a player must learn to overcome. In fact, much of what makes a game enjoyable in the first place comes from the mastery a player needs to develop to overcome obstacles in a game and the corresponding boost in self-efficacy (cf. Klimmt and Hartmann [6]). Given this combination of engagement and learning, it is therefore unsurprising that games used to teach people nontrivial instructional material, so called serious games, are garnering more and more popular attention; however the results of serious games in terms of learning goals so far have been mixed [23], [24].

We hypothesize that an important reason why serious games sometimes fail to reach their learning goals, is because games offer a rich multimodal and thereby cognitively demanding experience. As a person's working memory only has a limited capacity (cf. Paas, Renkl and Sweller [13]), and integrating new sensory data into a mental model happens within the boundaries of this working memory [10], such a rich experience may be too cognitively demanding, resulting in incorrect mental models of the instructional material, as was evidenced by Nelson and Erlandson [12]. Finding ways to optimize cognitive load during the design of the game is therefore an important step in creating effective and instructionally sound games (cf. Kiili [5]).

\section{ATtENTIONAL CUEING}

A number of ways in which the cognitive load in games may be mitigated have been proposed by, among others,
Moreno and Mayer [11], and Van der Spek, Wouters and Van Oostendorp [20]. Here, we will focus on using cues in order to guide a player's attention to the relevant information items at specific moments occurring in a game.

The rationale behind using cues is that sometimes a player may not know where to look for the information that is relevant to understanding the underlying instructional principles. This is paramount when the relevant information is less salient than irrelevant information, especially for novices who do not yet know how to distinguish relevant from irrelevant material [9], [22], or when the cognitive faculties of the player are overloaded by too much information at the same time to reliably separate the two [7]. As this visual search for relevant instructional material is for a part extraneous, and thus unnecessary, cognitive load, one generally wants to limit this as much as possible. Attentional cues then, are a way of guiding the attention of the player to the relevant material, or away from the irrelevant material, by means of subtle sensory stimuli.

Attentional cues are a natural fit for gaming and are starting to become widely applied, for instance in Mirror's Edge (EA/DICE) where the process of wayfinding is sped up by coloring the objects one has to run towards bright red, or in Fable 2 (Lionhead/Microsoft Game Studios) where non playable characters (NPCs) emit a colored halo as a way of communicating to the player which NPCs are friendly and which hostile. These visual cues intuitively work well, but scientific evidence of their value remains scarce. In the case of cueing wayfinding, Steiner and Voruganti [17] compared a number of different techniques to guide a player, who was instructed to simply explore the surroundings, to a target in a virtual environment. They distinguished between and compared: verbal cues, i.e. an NPC giving directions; written cues, i.e. signposts; a building landmark, i.e. a far off tower; environmental landmarks, i.e. fire and a path landmark, i.e. a glowing trail. All of these led the players to where the experimenters intended, but the glowing trail was significantly better in doing this. Conversely, the glowing trail and the NPC giving directions were found to be the most explicit means of guiding, whereas the tower and fire were mostly not perceived as guidance at all. Interestingly, and perhaps as a consequence of this study, Fable 2 also employs a glowing trail (of what they call 'breadcrumbs') to guide a player to the next important spot in a given quest.

Two caveats to the previous examples of guidance cues should be given however. Firstly, while cues are regularly used

This research has been supported by the GATE project, funded by the Netherlands Organization for Scientific Research (NWO) and the Netherlands ICT Research and Innovation Authority (ICT Regie). 
in games to nudge a player in a certain direction, it is unclear how they would fare in the context of learning. Do cues, for instance, relieve the burden and thereby stimulate deeper processing, are they only useful in dictating and automating certain procedural patterns, or will they even inhibit deeper processing and actually make the player a lazy learner? A number of experiments done on the effect of cueing in multimedia learning other than games, support the notion of cueing reducing cognitive load and benefiting learning (cf. Jeung, Chandler \& Sweller, [2]; Kalyuga, Chandler \& Sweller, [4]). Additionally, although they did not find that cueing reduced cognitive load, De Koning, Tabbers, Rikers and Paas [1] reported that, not only did cueing help in learning the cued material in the context of learning from an animation, but even improved learning of the uncued material. However it should be noted that these results are not uncontroversial, as Tabbers, Martens \& Van Merrienboer [18] only found significantly positive results on retention tests, and too much guidance in a problem solving context can even lead to lower learning gains [21].

Secondly, many games aspire to induce a sense of presence, the sense of psychologically being inside the virtual environment (cf. Slater \& Wilbur, [16]). The level of presenceinducing qualities of a game may be linked to the enjoyment of a video game [19], and while research on whether it influences task performance is still inconclusive, presence is imperative for games that have to induce stress, for example for phobiaalleviation [14]. A brightly colored halo around an NPC, or a glowing trail, as in the examples above, may certainly be effective, but it's not very realistic, and could therefore harm the immersive qualities of a game. In addition, games and their players can be quite competitive and some people may not want to be helped. As Steiner and Voruganti [17] showed that subtle cueing can also be effective, we therefore contend that guiding the player into the right learning direction, at least when it comes to games, should be done subtly and the effect on the engagement of the game should always be tested.

\section{HYPOTHESES}

Given the aforementioned considerations, we want to experimentally test the effect of subtle cues on learning gains from a serious game. We chose to use auditory cues, as these can be implemented in most games relatively easily and don't rely on using visual 'impossibilities' (such as the aforementioned halo) to get the player's attention. This leads us to our main hypothesis:

Providing auditory cues improves learning from a serious game, while it doesn't affect the engaging qualities of the game

Where improved learning is defined as performing significantly better on knowledge tests than a control group and also having a mental model that is closer to a referent mental model. Closely related to this, we additionally hypothesize that:

Providing auditory cues reduces the reliance of players on additional, flow-breaking help material

Where by flow-breaking help material we mean using the procedure button to see the triage procedure flowchart (to be explained here below in section 4, Code Red Triage).

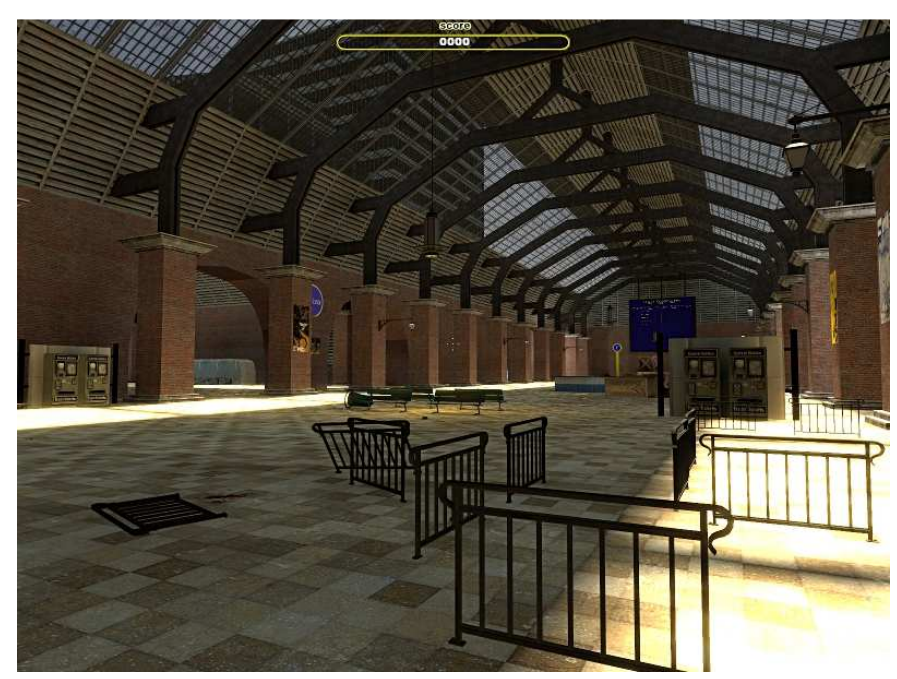

Figure 1 trainstation in game

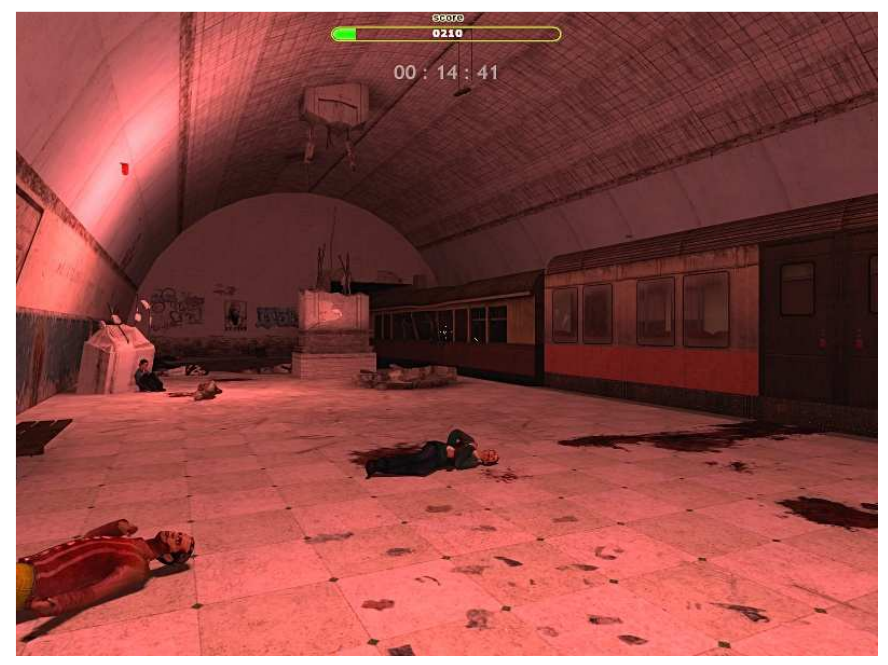

Figure 2 subway platform in game

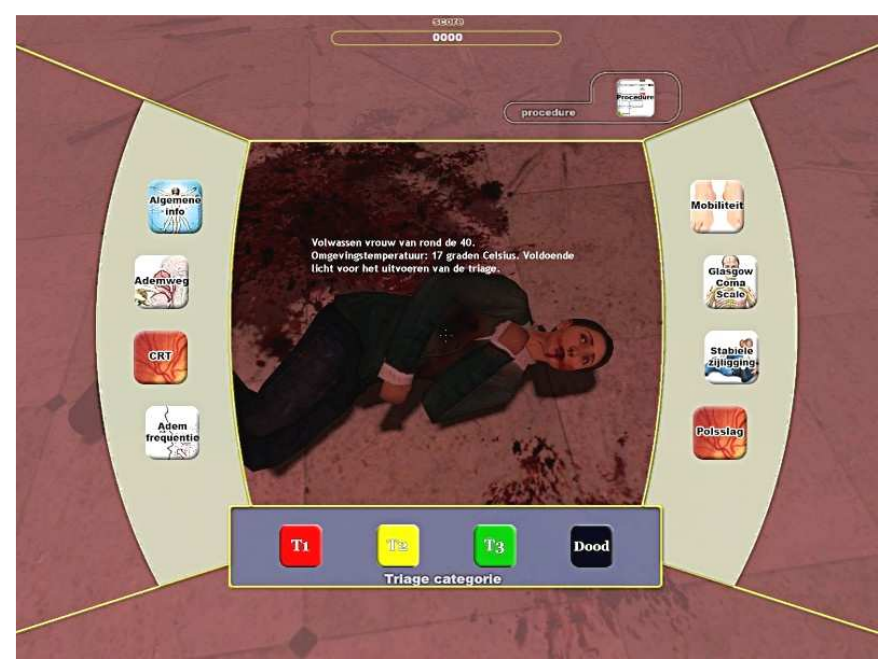

Figure 3 triage procedure in game 


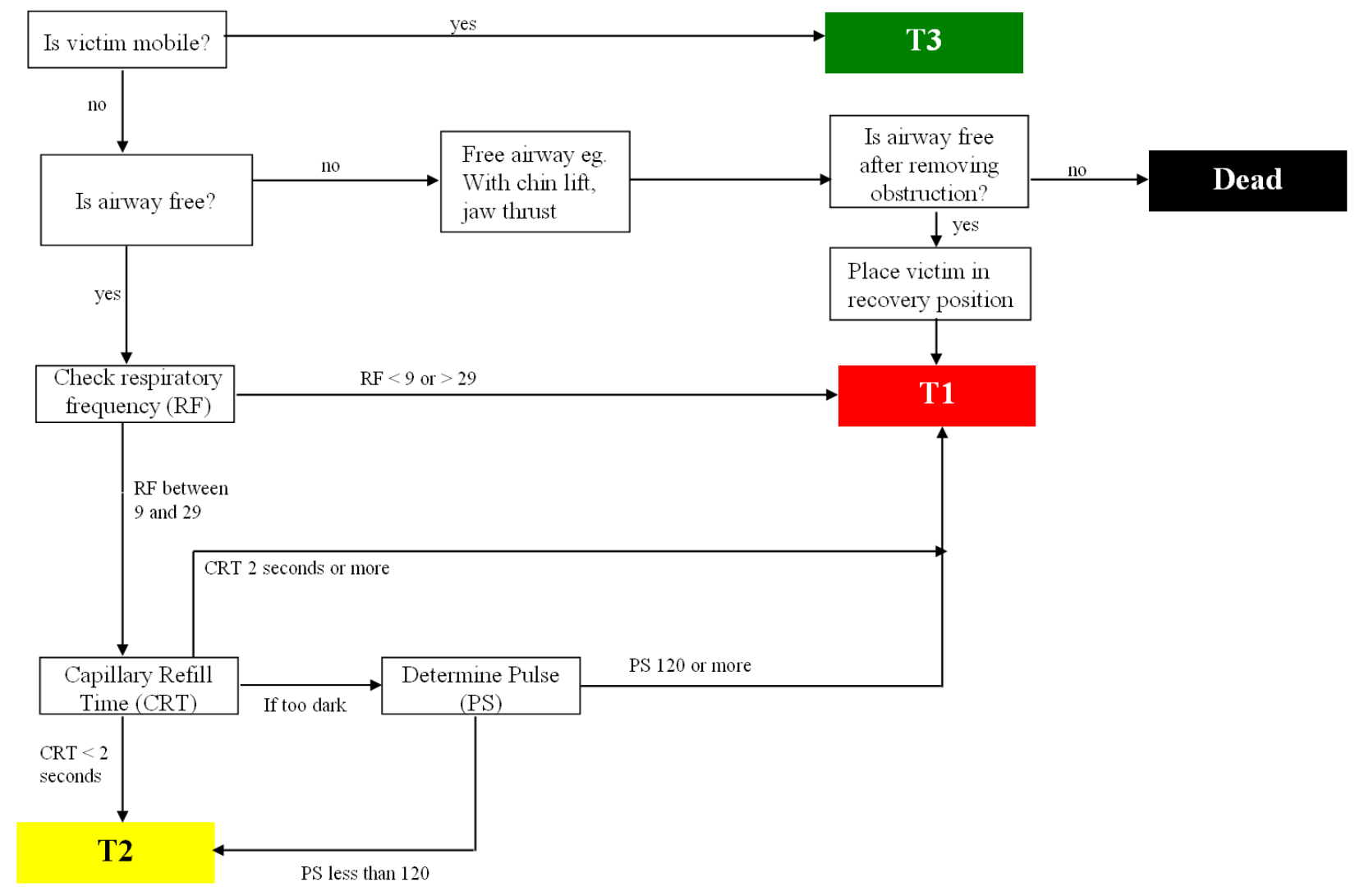

Figure 4 Triage flowchart

\section{Code Red Triage}

In order to test whether cueing improves learning gains from a serious game, we created a 3D first-person game, Code Red Triage, which trains players in performing a primary (sieve) triage, a technique for medical first responders to swiftly categorize the victims of a mass casualty event in order of urgency of needed medical attention [20].

The game starts in a train station (Figure 1), where players learn of a terrorist strike in the adjacent subway station. They have to find the way to subway platform number two and, upon arrival (Figure 2), categorize every victim according to the procedure of the primary triage. This procedure is relatively straightforward: first a player has to check whether the victim can still walk, secondly whether its airway is free, thirdly its respiratory frequency and lastly its blood circulation via the capillary refill time. Depending on the outcome of each of these steps, a victim can be classified in one of four triage categories: T3 (green or non-urgent), T2 (yellow or moderately urgent), T1 (red or highly urgent) and Dead (black; death imminent or already dead). Additionally, if the airway is blocked, players have to perform either a chin lift or a jaw thrust and, depending on the outcome, possibly put the victim in the stable recovery position. Furthermore, if it is too dark for the capillary refill time (which happens in a corridor, at the back of the platform and inside the subway train), the player has to check the heart rate via the pulse.

The procedure in the game is performed by means of a menu, with eight buttons portraying necessary, as well as irrelevant, steps, the resultant information in the middle and the triage categories at the bottom (Figure 3). A flowchart of the triage procedure can be seen in Figure 4. As we wanted the participants to get as little prior training or information as possible, so that the attentional cues optimally influenced the learning of the triage procedure we decided to provide the flowchart not before playing the game, but to make it inspectable during the game when needed. Players can access it via the button at the top right of the triage procedure screen (Figure 3).

\section{MENTAL MODELS}

In order to elicit the way in which information is stored in the mental model of the participants of our experiment, we use Pathfinder software [15]. Pathfinder is essentially a computer program that takes a set of concepts and gives these concepts back to the user as a randomized set of pairs. The user has to rate each pair of concepts on how much he or she thinks they are related, and from the resulting matrix of interrelatedness, Pathfinder draws so-called associative networks. These associative networks are (un)directed graphs, that give a 
pictorial view of how information is represented in the cognitive structure of the participant, and can be compared to another by means of a similarity measure. By calculating the similarity measure of a participant's mental model to a referent 'correct' mental model it is thus possible to effectively calculate how much a participant's mental model has improved (or deteriorated) after playing our game. The 'correct' referent mental model we created can be seen in Figure 5.

It is only a subset of the total triage procedure; in earlier tests (cf. Van der Spek et al. [20]) we had a larger portion of the procedure, but the number of combinations explodes rapidly with every added concept, making it tiresome for the participant and therefore unreliable. We furthermore deliberately chose to include conceptual items as well as procedural items. If these are connected to each other one gets what Kahler [3] calls strategic knowledge, and it gives a further indication of how robust the mental model is to when the participant encounters other related problems.

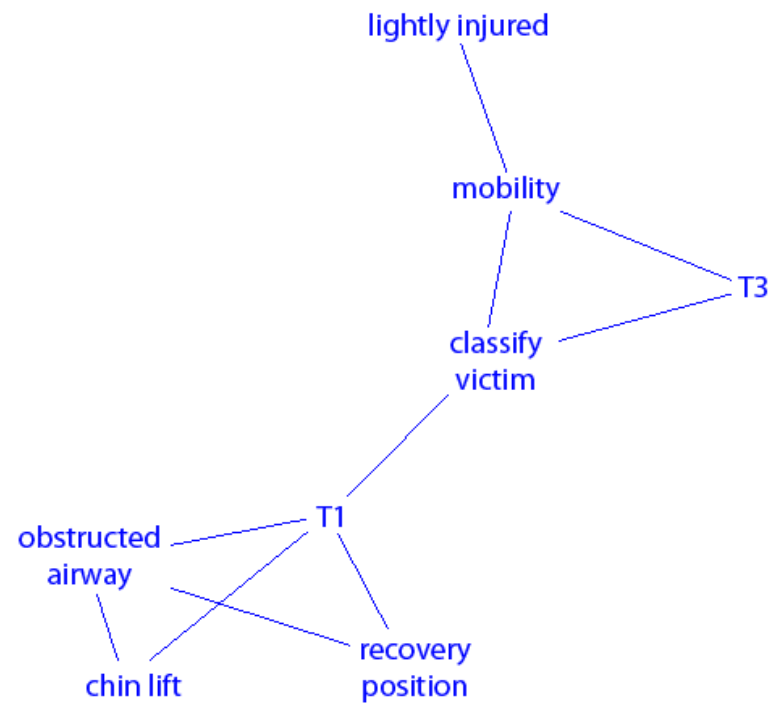

Figure 5 referent mental model

\section{METHOD}

\section{A. Experimental conditions}

The purpose of our experiment was to see if it was possible to guide a player in choosing and learning the correct procedure. One could do this rather explicitly, for instance by highlighting the correct button, but as we discussed previously, such explicit visual indicators can potentially harm the immersion into the game. As Steiner and Voruganti [17] showed that subtle cues can also be successful, we therefore wanted to test if we could cue a correct procedure with sounds that would not seem out of place in such a setting. In our case these sounds were: a footsteps sound to cue checking the mobility of a victim, a snoring sound to cue checking the airway, a fast breathing sound to cue the respiratory rate and a beating heart sound to cue checking the capillary refill time or pulse.
The experiment had two different versions of the game, one control condition with no auditory cues, and one cueing condition with the aforementioned auditory cues. Both had background music and other sounds, such as screaming, but the moment the player walked up to a victim and pressed the ebutton to commence the triage procedure, this sound would be automatically turned down in both conditions to a barely audible level. In the cueing condition, three seconds after the procedure was started, and roughly around the time the player would have read the introductory victim information, the footsteps sound was played. If the procedure should end there, for instance when the victim is still able to walk and is therefore deemed to be lightly injured, no further cueing sounds were played. If on the other hand another step in the procedure should be taken, for all following procedure steps three seconds after the last correct button was pressed, the cue for the next correct button was then played.

\section{B. Participants}

A total of 21 persons, mostly university students from different studies, participated in this experiment. Mean age was $21.48(S D=1.21), 11$ participants were randomly assigned to the cueing condition, with 5 male and 6 female, and 10 participants were randomly assigned to the control condition, with 6 male and 4 female. A total of 2 participants called themselves an avid gamer, 8 a casual gamer and 11 deemed themselves a non-gamer.

\section{Apparatus}

The game was made with the Source SDK, a development kit created and distributed freely by VALVe Software alongside The Orange Box (2007), for the creation of modifications ('mods') of Half Life 2 and other games that run on the Source engine. The game was played on a Dell XPS M1730 gaming laptop, with a 17" widescreen monitor, a dual GeForce 8800GTX 3D card and with a large Sony headset. The game ran smoothly on maximum detail and lighting settings with no hiccups in the framerate and only some minor (1 second or less) lag between pressing the triage procedure button and the corresponding menu to pop up. The laptop was set up in a closed laboratory room, with a movable screen on all sides to ensure a minimum of environmental distraction.

\section{Procedure}

The participants were sat in the enclosed space behind the computer and started by filling in a short demographics questionnaire. The first test was (1) the Pathfinder exercise to gauge their mental model, after which (2) they answered a ten item multiple choice paper questionnaire with combined conceptual and procedural questions. Next they received some basic instruction about how to navigate through the game environment. In line with other cueing studies (cf. Steiner and Voruganti [17]) no information was provided regarding the auditory cues.

After this the lights were turned off and they were allowed to play the game. There was no time limit on playing the game until they reached the subway platform, this was done to make the player acquainted with the controls and to measure how fast they could navigate through a $3 \mathrm{~d}$ environment, to provide us 
some indication of gaming proficiency. Upon reaching the platform, a visible timer counted down from 17 minutes, to give the player a sense of urgency; after 17 minutes the game ended. All but 2 participants found all 19 victims. Within the game we tracked the score of the player, which was fed back to the player via a progress bar at the top of the screen. Every victim classified correctly could award the player a score of 100 points (for a total of 1900 points), but points were deducted from this score for every essential step that was forgotten, taken in the wrong order, or steps that shouldn't have been taken. Additionally, up to 40 points time penalty were deducted; one point for every second longer than a preset time per victim. The (3) total score would then give us an accurate depiction of how well the players performed in the game.

When the game was finished, the lights were switched on again and the participants immediately had to fill in (4) the engagement questionnaire. For the engagement questionnaire, we used a subset of the ITC-SOPI questionnaire [8], a Likertlike questionnaire that's specifically designed to measure all types of presence that any multimedia setting can evoke. One of these types of presence is strongly related to engagement, resulting in a good mix of presence and engagement related questions (e.g. "I would have liked the game to go on longer"). The other factors that constitute presence are 'sense of physical space', 'ecological validity', and 'negative effects'.

After this the same procedure was followed as before the game: first (5) the Pathfinder test and then (6) the paper test, but with the items in a different order. On top of this we gave the participants (7) eight victim case descriptions with strictly procedural questions (i.e. given this victim, at this moment in the procedure what is the next step in the primary triage procedure), four of which were verbal, and four of which were pictorial, as in the game.

\section{RESULTS}

Both conditions significantly improved on their paper tests: from $M=3.2$ to $M=7.1, t(21)=-9.2, p<0.001$; as well as on their mental model similarity to the referent structure: from $M$ $=0.033$ to $M=0.232, t(21)=-5.5, \mathrm{p}<0.001$. However, the effect of cueing on these scores was decidedly less conclusive; the means and standard deviations for the tests are depicted in Table 1, we will go into more detail here.

Paper pre and posttest. While the participants had been randomly assigned to the groups, a small but significant difference unfortunately appeared in the pretest scores $(t(19)=$ -2.37, $p<0.05$ ), therefore the pretest score was added as a covariate. An ANCOVA with condition (control vs. cueing) as fixed factor, the paper posttest as dependent variable and the paper pretest as covariate did not yield any significant effect, meaning that cueing the correct procedure did not lead to a significantly better performance $(\mathrm{F}(1,18)=1.08, p=0.31)$.

In-game score. The in-game score between the two groups was also not significantly different $(\mathrm{F}(1,19)=0.01, p=0.94)$. Upon closer inspection, this was probably because every participant pressed the procedure button frequently, and followed the exact procedure as was outlined there. In fact, the number of times a participant pressed the procedure button was a much higher, and statistically significant, predictor of the score than the condition variable: $\mathrm{b}=0.61, t(18)=3.1, p<0.01$ (see Figure 6) versus $\mathrm{b}=0.15, t(18)=0.77, p=0.45$,

Use of in-game help. Our secondary hypothesis was that cueing helps in decreasing the amount of times a person presses the procedure button, and thereby increases the engagement/presence of the game. However, even though there seems to be a trend that the number of procedure button presses decreases because of cueing $(M=27.7, S D=8.9$ for control condition; $M=22.7, S D=9.0$ for cueing condition), this result is not significant (One-way ANOVA: $\mathrm{F}(1,18)=1.63, p=0.22$ ). The difference between groups in average time per victim was also not significant $(\mathrm{F}(1,19)=0.27, p=0.60)$, nor were the results of the ITC-SOPI engagement questionnaire $(\mathrm{F}(1,19)=$ $1.4, p=0.25)$. This means that, even though the cues didn't help in the game, they also didn't negatively affect the engagement.

\begin{tabular}{|l|l|c|c|c|c|}
\hline \multicolumn{2}{|c|}{ variable } & \multicolumn{2}{|c|}{ Control } & \multicolumn{2}{c|}{ Cueing } \\
\cline { 2 - 6 } \multicolumn{2}{|l|}{} & $\boldsymbol{M}$ & $\boldsymbol{S D}$ & $\boldsymbol{M}$ & \multicolumn{1}{c|}{ SD } \\
\hline $\begin{array}{l}\text { Paper pre-post } \\
\text { test }\end{array}$ & before & 2.60 & 0.97 & 3.73 & 1.19 \\
\hline $\begin{array}{l}\text { Mental model } \\
\text { similarity }\end{array}$ & after & 7.40 & 1.84 & 6.82 & 0.054 \\
\hline $\begin{array}{l}\text { In-game score } \\
\text { after }\end{array}$ & 0.027 & 0.103 & 0.039 & 0.119 \\
\hline $\begin{array}{l}\text { ITC-SOPI } \\
\text { Engagement }\end{array}$ & & 1130.0 & 0.141 & 0.153 & 252.8 \\
\hline $\begin{array}{l}\text { No. of times } \\
\text { procedure } \\
\text { checked }\end{array}$ & & 3.73 & 267.4 & 1121.1 & 0.47 \\
\hline
\end{tabular}

Table 1 results of the cueing experiment 
Mental models. The only between group performance measure that showed a significant difference, is the mental model measure. An ANCOVA with cueing as fixed factor, the similarity measure after the game as dependent variable and the similarity before playing the game as covariate, yields $\mathrm{F}(1,18)$ $=8.40, p<0.01$. This holds if the number of times the procedure is checked is added as a covariate as well $(\mathrm{F}(1,17)=$ $5.17, p<0.05)$. Contrary to our hypothesis, the cueing condition performed worse than the control condition (see Figure 7), meaning that guiding the player during learning in a serious game by means of auditory cues actually worsens mental model construction.

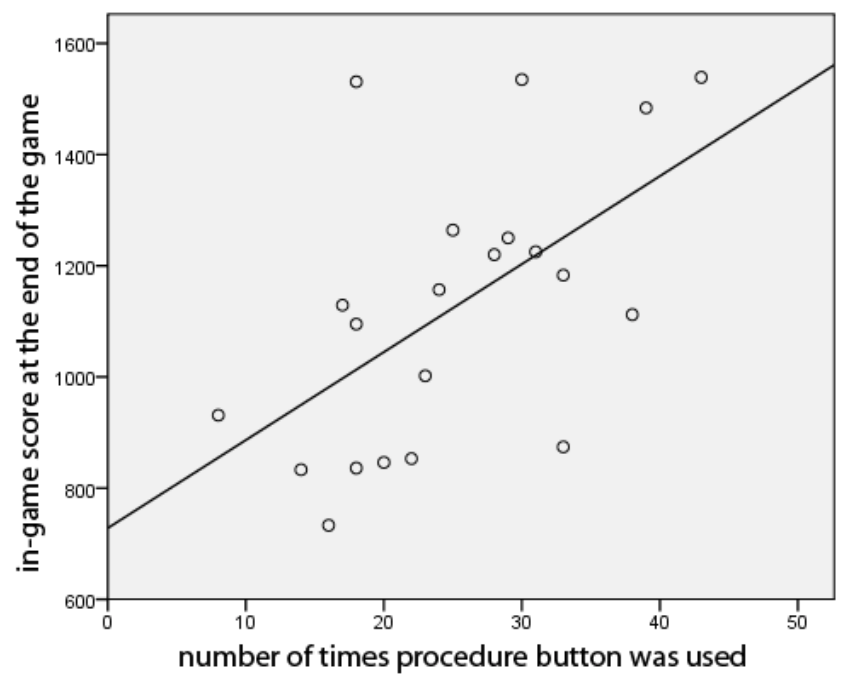

Figure 6 triage procedure flowchart usage as predictor for in-game score

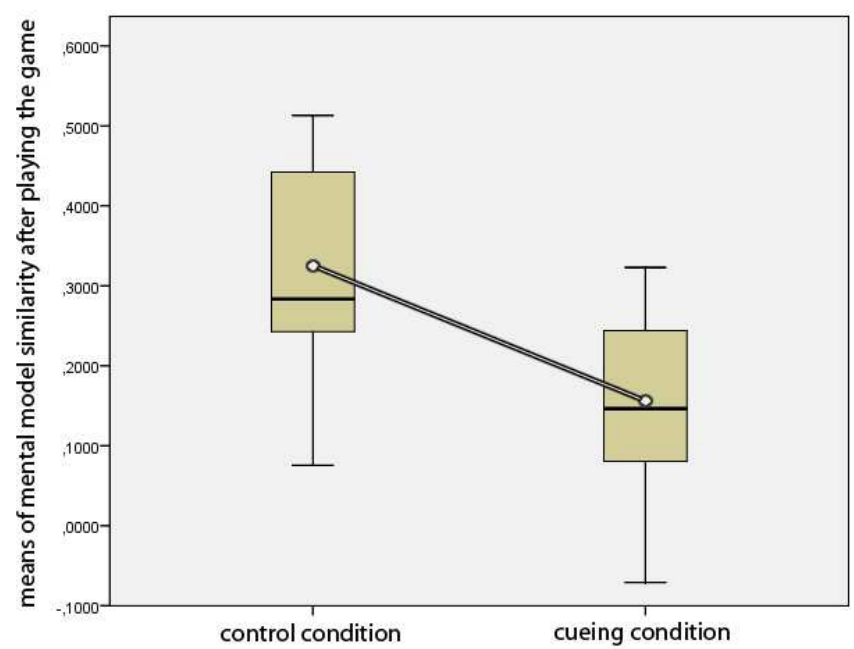

Figure 7 boxplot of mental model similarity means with ANCOVA result superimposed
Procedural cases. The procedural questionnaire at the end (verbal and visual items) also yielded no significant difference between the groups, but, as an aside, do give an interesting insight. Of all the tests (paper pre-posttest, mental model similarity, in-game score, procedural questions with victim cases) used to measure practically the same thing: a participant's knowledge of the triage procedure, none were highly correlated with each other, except for a very strong correlation between the in-game score and the procedural questions with a picture from the game: $r(21)=0.71, p<$ 0.001 . This indicates that when one wants to test how well someone performed in a game, it is best to use pictures from the game, but also, at least in our case, that the in-game score is a relevant measure of how well the triage procedure is performed by the player. In addition, a slightly weaker (and perhaps more trivial) correlation between the verbal and visual procedure questions was found $(r(21)=0.52, p<0.05)$.

\section{CONCLUSION AND DISCUSSION}

The straightforward conclusion that can be drawn from our experiment is that auditory cues alone, as implemented in our experiment, do not suffice in helping the player to correctly learn a procedural task in a serious game. Contrarily so, the mental model construction is even negatively affected, and therefore possibly also the transfer to other settings. No significant differences were found in the in-game score or paper posttests, meaning that the participants probably hardly noticed or understood the auditory cues. This could indicate that in cases where the player has to learn a certain technique, the more explicit visual cues as used in contemporary videogames, while realism-breaking, may in fact be necessary in order to have the player notice them. Steiner and Voruganti [17] showed that subtle cues can guide the player to visit a designated spot in, it should be noted, a rather sparse virtual environment (especially compared to contemporary commercial games). But they also discovered that a trail of strangely glowing rocks worked the best. With more and more visual clutter in a game world, made possible by ever increasing technical prowess, it may well be the out-of-place realism-breaking visual details that are needed to sufficiently draw our attention. More future research is needed to satisfactorily determine this.

The less straightforward conclusion pertains to the fact that, even though we did an extensive pilot beforehand (cf. Van der Spek et al. [20]), a number of threats to the validity of the results can be discerned. First and foremost the small number of participants; ten participants per group may be too little to accurately extricate the effects of such a subtle cueing mechanism. With more participants, some of the inconclusive results, for example the number of times the procedure flowchart was consulted, could become clearer.

In addition, when the experiment was set up, we were expecting to see either a positive result or no result of the auditory cues and therefore, given the paucity of the participants at hand, chose for a condition with no cues and a condition with auditory cues. Mental model construction clearly suffered from the cues, but it is unclear whether the 
guidance during the procedure made the player 'lazy', as in Van Nimwegen and Van Oostendorp [21], or whether the cues weren't recognized (consciously or unconsciously) as such and only distracted the player during mental model construction.

Another problem with our experimental setup was that only the main steps in the triage procedure were cued: mobility, airway, respiratory rate and blood circulation. While these make up the most important parts of the procedure, other decisions, such as performing a chin lift or jaw thrust and placing a victim in the recovery position, or the correct values accompanying the heart or respiratory rate were not cued (see the triage flow chart in Figure 4). As De Koning et al. found that cueing also improved learning of the uncued materials, we wanted to see if the same thing would happen in our game as well, and therefore these uncued items were also part of our ingame score and the pre and posttests. However, as cueing did not improve learning in general, this additional step may in retrospect have been slightly overzealous, where we should have focused only on the main effect of cueing. In fact, when we look at the paper ten-item questionnaire, only four of the questions would have directly benefited from our auditory cues. If we then take only these four questions, and do an exploratory ANCOVA with the pretest and the number of times the procedure button was clicked as covariates, we actually see a significant and positive effect of auditory cues on knowledge acquisition $\mathrm{F}(1,17)=5.17, p<0.05$. This would imply that cueing does in fact have a beneficial effect on learning in a serious game, but only in a very specific way. However it is highly questionable whether only four questions have enough discriminative power for such a claim, so we have to examine this issue more closely in further research.

\section{REFERENCES}

[1] B.B. De Koning, H.K. Tabbers, R.M.J.P. Rikers, and F. Paas, "Attention Cueing as a Means to Enhance Learning from an Animation". Applied Cognitive Psychology, vol. 21, pp. 731-746. 2007.

[2] H. Jeung, P. Chandler, and J. Sweller, "The role of visual indicators in dual sensory mode instruction". Educational Psychology, vol. 17(3), pp. 329-343. 1997.

[3] S. Kahler, "A comparison of knowledge acquisition methods for the elicitation of procedural mental models". (Doctoral dissertation). 2001. Last retrieved 15 nov. 2009, from URL http://www.lib.ncsu.edu/theses/available/etd-12132002105613/unrestricted/etd.pdf

[4] S. Kalyuga, P. Chandler, and J. Sweller, "Managing split-attention and redundancy in multimedia instruction". Applied Cognitive Psychology, vol. 13, pp. 351-371. 1999.

[5] K. Kiili, "Digital game-based learning: Towards and experiential gaming model”. The Internet and Higher Education, vol. 8(1), pp. 13-24. 2004.

[6] C. Klimmt, and T. Hartmann, "Effectance, Self-Efficacy, and the Motivation to Play Video Games". In P. Vorderer \& J. Bryant (Eds.), Playing Video Games, Motives, Responses, and Consequences (pp. 133145). Lawrence Erlbaum Associates, Inc. Mahwah, NJ. 2006.
[7] N. Lavie, "Distracted and confused?: Selective attention under load". TRENDS in Cognitive Science, vol. 9(2), pp. 75-82. 2005.

[8] J. Lessiter, J. Freeman, E. Keogh, and J. Davidoff, "A cross-media presence questionnaire: The ITC-Sense of Presence Inventory". Presence: Teleoperators \& Virtual Environments, vol. 10, 3, pp. 282297. 2001.

[9] K.R. Lowe, "Animation and learning: selective processing of information in dynamic graphics". Learning and Instruction, 13(2), pp. 157-176. 2003.

[10] R.E. Mayer, "Multimedia learning". London: Cambridge University Press. 2001.

[11] R. Moreno, and R.E. Mayer, "Interactive multimodal learning environments". Educational Psychology Review, vol. 19, pp. 309-326. 2007.

[12] B.C. Nelson, and B.E. Erlandson, "Managing cognitive load in educational multi-user virtual environments: reflection on design practice". Educational Technology Research and Development, vol. 56, pp. 619-641. 2007.

[13] F. Paas, A. Renkl, and J. Sweller, "Cognitive Load Theory and Instructional Design: Recent Developments". Educational Psychologist, 38(1), pp. 1-4. 2003.

[14] M.J. Schuemie, P. Van der Straaten, M. Krijn, and C.A.P.G. Van der Mast, "Research on Presence in Virtual Reality: A Survey". Cyberpsychology \& Behavior, vol. 4(2), pp. 183-201. 2001.

[15] R.W. Schvaneveldt, "Pathfinder associative networks: studies in knowledge organization”. Norwood, NJ: Ablex. 1990.

[16] M. Slater, and S. Wilbur, "A framework for immersive virtual environments (FIVE): Speculations on the role of presence in virtual environments". Presence: Teleoperators and Virtual Environments, vol. 6(6), pp. 603-616. 1997.

[17] K.E. Steiner, and L. Voruganti, "A comparison of guidance cues in desktop virtual environments". Virtual Reality, 7, 140-147. 2004.

[18] H.K. Tabbers, R.L. Martens, and J.J.G. Van Merrienboer, "Multimedia instructions and cognitive load theory: Effects of modality and cueing". British Journal of Educational Technology, vol. 74(1), 71-81.

[19] R. Tamborini, and P. Skalski, "The Role of Presence in the Experience of Electronic Games". In P. Vorderer \& J. Bryant (Eds.), Playing Video Games, Motives, Responses, and Consequences (pp. 225-240). Lawrence Erlbaum Associates, Inc. Mahwah, NJ. 2006.

[20] E.D. Van der Spek, P. Wouters, and H. Van Oostendorp, "Code Red: Triage. Or, COgnition-based DEsign Rules Enhancing Decisionmaking TRaining In A Game Environment". British Journal of Educational Technology, in press.

[21] C. Van Nimwegen, and H. Van Oostendorp, "The questionable impact of an assisting interface on performance in transfer situations". International Journal of Industrial Ergonomics, vol. 39(3), pp. 501-508. 2009.

[22] H. Van Oostendorp, M.J. Beijersbergen, and S. Solaimani, "Conditions for Learning from Animations". In Proceedings Conference of the Learning Sciences. Utrecht, Netherlands: Utrecht University. 2008.

[23] J.J. Vogel, D.S. Vogel, C.A. Cannon-Bowers, K. Muse, and M. Wright, "Computer gaming and interactive simulations for learning: a metaanalysis". Journal of Educational Computing Research, vol. 34, 229243. 2006.

[24] P. Wouters, E.D. Van der Spek, and H. Van Oostendorp, "Current practices in serious game research: a review from a learning outcomes perspective". In T. M. Connolly, M. Stansfield \& L. Boyle (Eds), Games-based learning advancements for multisensory human computer interfaces: techniques and effective practices (pp. 232-255). Hershey, PA: IGI Global. 2009. 